\title{
Use of preferentially replicating bacteria for the treatment of cancer
}

\author{
Mario Sznol, Stanley L. Lin, David Bermudes, Li-mou Zheng, and Ivan King
}

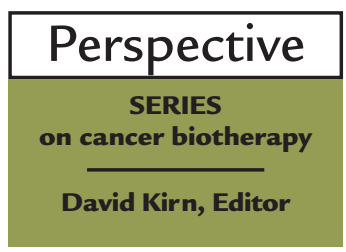

Vion Pharmaceuticals Inc., New Haven, Connecticut 06511, USA

Address correspondence to: Mario Sznol, Vion Pharmaceuticals Inc., 4 Science Park, New Haven, Connecticut 06511, USA.

Phone: (203) 498-4210; Fax: (203) 498-4211; E-mail: msznol@vionpharm.com.

The past several years have seen renewed interest in the treatment of cancer with live microorganisms, based on the observation that some microorganisms display selective replication or preferential accumulation in the tumor microenvironment. Preferential replication offers great potential to amplify the therapeutic effect of the microorganism while sparing normal tissues from toxicity. Much of the current research intended to achieve selective replication within, and lysis of, tumor cells has focused on viruses, but recent observations in murine models with facultative anaerobic bacteria (1), as well as data generated more than 30 years ago with obligate anaerobic bacteria (2), indicate that some bacterial species can also preferentially replicate and accumulate within tumors. In contrast to viruses, the bacteria reside primarily in the extracellular tumor microenvironment (3) and possess certain features that may be advantageous in the treatment of cancer. Thus, bacteria are motile, which facilitates their spread throughout the tumor and can help target systemic disease.

Because of their large genome size, bacteria can readily express multiple therapeutic transgenes, such as cytokines or pro-drug-converting enzymes, and their spread can be controlled with antibiotics if necessary.

Interestingly, the observation that conditions within experimental animal tumors could favor the preferential replication of bacteria, compared with normal tissues, was made approximately 40 years ago, when certain Clostridia species were shown to proliferate extensively in some tumors. Proliferation presumably occurred in the hypoxic and necrotic regions of the tumor, but not in well-oxygenated normal tissues (2). Clostridia-mediated tumor lysis was demonstrated primarily in the moreestablished or larger tumors, expected to contain regions of hypoxia. The Clostridia were often absent or had no discernible antitumor effect in small metastatic lesions. Tumor pathology resulting from Clostridia infection was manifested as a liquid necrotic center surrounded by a rim of viable tumor cells, from which the tumor would eventually regrow. Some animals became ill and died during the peak of oncolysis, presumably from the toxic and/or systemic inflammatory effects of bacteria, their byproducts, or necrotic tumor debris.

After similar observations regarding the preferential replication of Clostridia in tumors were made in rat and hamster models $(4,5)$, clinical trials were initiated with a non-toxin-producing strain of Clostridia spores (6). Patients with advanced cancer typically developed fever and positive blood cultures for approximately 1 week and occasionally required supportive care involving treatment with antibiotics. Most patients had no evidence of objective regression or change in tumors, but several patients with large tumor masses developed fluctuant abscesses within tumor masses, and cultures of the masses revealed the injected organism. Because of the overall lack of clinical efficacy, further studies with Clostridia spores were abandoned, but, in the past several years, investigators have begun to explore obligate anaerobic Clostridia in animal models as a means to deliver pro-drug-converting enzymes and other gene-based anti-cancer agents specifically to tumors $(7,8)$.

Preclinical and clinical studies with Clostridia have identified several obstacles to the development of preferentially replicating bacteria for cancer biotherapy. Successful clinical candidates should infect and multiply within both small and large metastatic lesions, should be attenuated so that they induce minimal systemic inflammatory responses, should replicate in tumors without causing an excessive inflammatory reaction that could lead to formation of toxic pustular abscesses, and, to provide an additional margin of safety, should be sensitive to common antibiotics.

In 1997, Pawelek et al. reported that Salmonella would infect and preferentially accumulate within implanted tumors in mice, achieving tumor/normal tissue ratios of approximately 1,000:1 (1). This observation, combined with ease of manipulation, suggested that Salmonella might be developed as a clinically useful anti-cancer agent. Wild-type Salmonella, and in particular Salmonella typhimurium, produces self-limited enteritis in most healthy adults, infects both mice and humans, and can easily be manipulated to carry foreign genes. This species also exists as a facultative anaerobe, allowing it to survive in both oxygenated and hypoxic conditions, so it would be expected to colonize small metastatic lesions as well as larger tumors.

A surprising observation in the preclinical development was the ability of attenuated Salmonella species to retard tumor growth in a broad range of staged syngeneic and xenograft murine tumor models (Table 1). Thus, when single doses of the Salmonella were administered intravenously at the time tumors were $100-400 \mathrm{mg}$ in size (1, 
Table 1

Effect of attenuated Salmonella typhimurium on growth of tumor expressed as tumor volume doubling time (TVDT) measured in days
Tumor

B16-F10 melanoma

LOX human melanoma

DLD1 human colon

Late stage

Early stage

WiDr colon

A549 lung

MDA-MB-231

Breast carcinoma

HTB177

Lung carcinoma

AP $=<0.01$ versus vehicles
TVDT (d)

2.74

$4.32^{\mathrm{A}}$

$6.06^{\mathrm{A}}$

$9.25^{\mathrm{A}}$

1.98

$16.48^{\mathrm{A}}$

6.02

NA

$14.32^{\mathrm{A}}$

6.84

$14.53^{\mathrm{A}}$

9.66

$21.52^{\mathrm{A}}$

5.53

$15.69^{\mathrm{A}}$

2.17
VNP20009 $1 \times 10^{6} \mathrm{CFU} \quad 7.79^{\mathrm{A}}$

$9,10)$ or, in the case of metastatic models, after small lung metastases were established (11), tumor growth was inhibited in most models for prolonged periods, in some cases up to 50-60 days. Several of the models showed improved survival times, but tumors eventually resumed growth, resulting in the death of the animals. The tumor site-related and systemic toxicities reported in preclinical studies of strictly anaerobic bacteria were not observed, although after a prolonged period, some subcutaneous tumors developed a central necrotic region.

\section{Engineering Salmonella as a biotherapeutic agent}

To develop a clinical candidate with a high safety profile, a wild-type strain of Salmonella typhimurium was attenuated by partial deletion of the $m s b B$ gene, whose product is responsible for addition of a terminal myristic acid group in the formation of lipid A (12). Lipopolysaccharide derived from these lipid A mutants is markedly diminished in ability to induce TNF- $\alpha$ in vitro in human monocytes and in vivo after administration to mice and pigs (9). As an additional safeguard, deletion of the purI gene (requirement for an external source of purine, e.g., adenine) was engineered into the $m s b B^{-}$Salmonella strain as a second attenuating mutation. The auxotrophic lipid A mutants could be administered intravenously at single doses of up to $5 \times 10^{7}$ $\mathrm{cfu} / \mathrm{kg}$ to naive, immune-competent mice without significant toxicity. The gene modifications did not affect the ability to achieve high tumor/normal tissue ratios in mouse models (Table 2), and the bacteria maintained their capacity to inhibit the growth of both subcutaneous tumors and lung metastatic disease.

Further characterization of the attenuated Salmonella revealed that nude and beige mice were between 5- to 10fold less tolerant of the attenuated Salmonella, and severe-combined immunodeficient (SCID) mice were approximately 50-fold less tolerant compared with immune-competent mice, which was consistent with the enhanced sensitivity of SCID mice to Salmonella infection previously reported with other attenuated Salmonella strains (13). Nevertheless, Salmonella-mediated mortality in SCID mice could be completely prevented without diminishing antitumor efficacy by administering antibiotics. Pigs and monkeys were even less sensitive to the toxicity of attenuated Salmonella than mice, with monkeys tolerating intravenous doses of up to $2.5 \times 10^{9}$ $\mathrm{cfu} / \mathrm{kg}(10)$. In response to administration of a clinical strain of attenuated Salmonella, monkeys demonstrated only minor and reversible transaminase elevations, and mild and rapidly reversible hematologic toxicity.

The kinetics of colonization of various tissues, examined in both mice and monkeys, provide some insight into to the mechanism of preferential accumulation of bacteria in tumors. Two hours after administration of 1 $\times 10^{6} \mathrm{cfu} /$ mouse, attenuated Salmonella accumulated predominantly in liver and spleen. By 24 hours, bacterial tissue levels remained highest in both spleen and liver, but increased in other tissues such that levels ranged from 1 $\times 10^{4}$ to $2 \times 10^{4} \mathrm{cfu} / \mathrm{g}$ in kidneys and lung to $2 \times 10^{5}$ and $9 \times 10^{5} \mathrm{cfu} / \mathrm{g}$ in liver and spleen, respectively. At neither time were significant levels detected in brain. In tumorbearing mice, bacterial levels in tumors uniformly exceeded $1 \times 10^{8} \mathrm{cfu} / \mathrm{g}$ tumor, regardless of tumor type or host, as demonstrated in syngeneic mouse tumors, spontaneous mouse tumors, and human tumor xenografts. Intratumoral bacterial levels persisted for greater than 4 weeks. In healthy monkeys, bacteria were shown to clear from circulation and most organ sites within days, but persisted at low levels in liver and spleen for 30-40 days before being fully eliminated from the body. Bacteria recovered from animals were genetically stable with regard to 4 specific markers and were unchanged in sensitivity to most common antibiotics.

The mechanisms responsible for the initial infection of tumor, followed by preferential accumulation of bacteria to high levels, compared with normal tissues, are not completely understood. Differences between tumor and normal vasculature and blood flow patterns could be involved in favoring entry into and entrapment of bacteria within tumors. Similarly, bacteria may be delivered to tumor by macrophages or monocytes, or per-

\section{Table 2}

Solid tumor targeting of attenuated Salmonella typhimurium

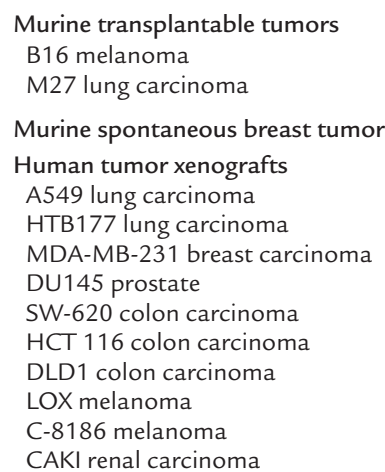

Murine transplantable tumors

B16 melanoma

M27 lung carcinoma

Murine spontaneous breast tumor

Human tumor xenografts

A549 lung carcinoma

HTB177 lung carcinoma

MDA-MB-231 breast carcinoma

DU145 prostate

SW-620 colon carcinoma

HCT 116 colon carcinoma

DLD1 colon carcinoma

LOX melanoma

C-8186 melanoma

CAKI renal carcinoma

Tumor: liver ${ }^{A}$ 12,000:1 10,000:1

700:1

$300: 1$

$$
\begin{array}{r}
4,000: 1 \\
34,000: 1 \\
24,000: 1 \\
275: 1 \\
17,000: 1 \\
15,000: 1 \\
3,000: 1 \\
1500: 1 \\
250: 1
\end{array}
$$

${ }^{A}$ Ratios of colony-forming units per gram of tissue. Liver represents normal tissue with highest level of accumulation. Ratios obtained days 2-4 after injection of bacteria. 
haps other cells invaded by bacteria. Regardless of the mechanism of entry, the data suggest that infection occurring from the bloodstream requires only a small number of organisms.

Studies in mice bearing colateral tumors also support the notion that low-level bacteremia can suffice to allow tumor colonization by Salmonella. In these animals, infection of a distal, contralateral tumor occurs after a 1week delay after intratumoral inoculation of the primary tumor, but at no time are bacteria detected in blood. These studies also demonstrated that colonization of a single tumor site could serve as a bacterial reservoir for eventual colonization at distal tumor sites. Furthermore, antibodies directed against Salmonella lipopolysaccharide components have been detected within 1 and 2 weeks after Salmonella administration in monkeys and mice, respectively. Even in the presence of a protective antibody response induced by killed wild-type Salmonella, we have found that the antitumor activity of attenuated Salmonella was preserved, suggesting that enhanced clearance did not affect the ability to colonize tumors and mediate an antitumor effect.

Because only a small number of organisms are required for colonization of tumor, factors present within the tumor microenvironment, perhaps interacting with specific characteristics of the bacteria, are more likely to contribute to the development of high tumor/normal tissue ratios. A nutrient-rich environment within the tumor could be a contributing factor to preferential bacterial replication. Furthermore, tumors grow rapidly, have substantial cell turnover and are likely to rapidly develop hypoxic and necrotic areas that distinguish the tumor environment from normal tissue. There is also substantial indirect evidence to suggest that bacterial clearance from tumors is slower and less effective than from normal tissues. Our data, demonstrating only a 5to 50 -fold decrease in tolerance to bacteria in nude or SCID relative to immunocompetent mice, suggest that $\mathrm{T}$-cell immunity plays, at most, a minor role in clearance of the attenuated bacteria. Bacterial clearance and suppression of virulence is mediated by multiple host defenses including granulocytes, macrophage-mediated phagocytosis, secretion of antibacterial peptides, antibodies, and elements of the complement system. Within tumors, granulocytes are rarely observed, suggesting a highly permissive environment for bacterial survival and replication. Secretion of TGF- $\beta$ or other immunosuppressive cytokines by tumor cells or stromal cells has been shown to inhibit granulocyte infiltration and activation (14). Recently, inhibitors of complement activation have been found on the surface of several tumor cell lines $(15,16)$. Finally, studies of tumor blood flow and pressure suggest the presence of substantial physical barriers to the entry of antibodies (17). Another unknown variable that could contribute to the ability of bacteria to enter and preferentially accumulate within tumors is the capacity of bacteria to invade tumor cells $(1,3)$.

Several mechanisms could be responsible for the broad antitumor activity of Salmonella observed in animal models, and consideration of these mechanisms is important for optimization of antitumor activity during clinical development. Despite the features designed into attenu- ated Salmonella to limit inflammatory responses and permit safe systemic administration, these bacteria still induce local inflammatory responses, perhaps by virtue of their capacity to accumulate to high levels in tumors and to reside there for long periods. Additional contributing factors to antitumor efficacy could include a type III secretion mechanism, by which bacteria may inject toxic peptides from the bacterial cytoplasm directly into the cytoplasm of mammalian cells (18); selective intratumoral intravascular coagulation and enhanced tumor encapsulation, perhaps as a consequence of cytokine induction within the tumor; the consumption or destruction of nutrients and growth factors within the tumor microenvironment; and production or secretion of toxins into the extracellular environment. Additional complexities arise from potential changes in bacterial replication and function that may depend on concentration of bacteria, residence time, and changing conditions within the tumor.

\section{Salmonella therapy in the clinic}

The attenuated, unarmed Salmonella typhimurium has recently entered clinical trials. Initial studies are designed for intratumoral administration, followed by studies of intravenous administration. The pattern of tumor growth inhibition observed in animal studies indicates that combinations with other anticancer agents would be desirable in the future, as the bacteria alone only delayed tumor growth, and tumor growth ultimately proceeded at the periphery of the tumor, despite the persistence of bacteria in these lesions. Particularly interesting will be strategies to enhance the local tumor inflammatory response induced by the attenuated bacteria. Current development of subsequent Salmonella has focused on delivery and expression of "therapeutic" genes, such as cytokines, pro-drug-converting enzymes, and agents toxic to tumor. Choice of the gene must be given careful consideration in order to produce agents that take full advantage of the large tumor/normal tissue ratios, and therefore would be likely to yield maximum patient benefit. Genes that produce maximum cytotoxic, proinflammatory or vascular destructive effects at the site of the tumor are to be preferred, providing that their products are not toxic to normal tissues, particularly liver, when present at 1,000-fold lower concentrations. Ideally, the secreted substance or activated drug should have a short circulating half-life and exert primarily paracrine antitumor effects. It is also desirable that the substance synergize with the antitumor effects of the bacteria and not affect the bacteria's persistence or replication in tumors. In the event that toxicities do evolve from production of the selected protein in the liver or from systemic release, it must be possible to terminate the bacteria and eliminate the toxicity, using antibiotics. Even under the latter circumstances, the tumor would be exposed to far greater concentrations of the agent than normal tissue, and presumably even a limited exposure might have substantial therapeutic benefit.

With the numerous effector genes that could be engineered into bacterial hosts, therapies could be extended to sequential or concurrent administration of similar or 
different bacteria that contain separate gene products. Demonstration of the central concept of selective intratumoral accumulation of bacteria in cancer patients can be expected to lead to a vast and novel repertoire of therapeutic options for the treatment of metastatic disease.

1. Pawelek, J.M., Low, K.B., and Bermudes, D. 1997. Tumor-targeted Salmonella as a novel anticancer vector. Cancer Res. 57:4537-4544.

2. Mose, J.R., and Mose, G. 1964. Oncolysis by Clostridia. I. Activity of Clostridium butyricum (M-55) and other nonpathogenic Clostridia against the Ehrlich carcinoma. Cancer Res. 24:212-216.

3. Kops, S.K., et al. 1997. Salmonella typhimurium as an anti-cancer vector: localization within solid tumors. Proc. Am. Assoc. Cancer Res. 38:7. (Abstr.)

4. Thiele, E.H., Arison, R.N., and Boxer, G.E. 1964. Oncolysis by Clostridia. III. Effects of Clostridia and chemotherapeutic agents on rodent tumors. Cancer Res. 24:222-231.

5. Engelbart, K., and Gericke, D. 1964. Oncolysis by Clostridia. V. Transplanted tumors of the hamster. Cancer Res. 24:239-243.

6. Carey, R.W., Holland, J.F., Whang, H.Y., Netter, E., and Bryant, B. 1967. Clostridial oncolysis in man. Eur. J. Cancer. 3:37-46.

7. Lemmon, M.J., et al. 1997. Anaerobic bacteria as a gene delivery system that is controlled by the tumor microenvironment. Gene Ther. 4:791-796.

8. Fox, M.E., et al. 1996. Anaerobic bacteria as a delivery system for cancer gene therapy: in vitro activation of 5-fluorocytosine by genetically engineered clostridia [erratum 1996, 3:741]. Gene Ther. 3:173-178.

9. Low, K.B., et al. 1999. Lipid A mutant Salmonella with suppressed viru- lence and TNFalpha induction retain tumor-targeting in vivo [see comments]. Nat. Biotechnol. 17:37-41.

10. Clairmont, C., et al. 1999. VNP20009, a genetically modified Salmonella typhimurium: anti-tumor efficacy, toxicology, and biodistribution in preclinical models. Clin. Cancer Res. 5(Suppl.):102. (Abstr.)

11. Zheng, L.M., et al. 1997. Attenuated Salmonella typhimurium inhibited tumor metastasis in vivo. Proc. Am. Assoc. Cancer Res. 38:9. (Abstr.)

12. Somerville, J.E., Jr., Cassiano, L., and Darveau, R.P. 1999. Escherichia coli msbB gene as a virulence factor and a therapeutic target. Infect. Immun. 67:6583-6590.

13. Guilloteau, L.A., Lax, A.J., MacIntyre, S., and Wallis, T.S. 1996. The Salmonella dublin virulence plasmid does not modulate early $\mathrm{T}$-cell responses in mice. Infect. Immun. 64:222-229.

14. Chen, J.J., Sun, Y., and Nabel, G.J. 1998. Regulation of the proinflammatory effects of Fas ligand (CD95L). Science. 282:1714-1717.

15. Schmitt, C.A., Schwaeble, W., Wittig, B.M., Meyer zum Buschenfelde, K.H., and Dippold, W.G. 1999. Expression and regulation by interferongamma of the membrane-bound complement regulators CD46 (MCP), CD55 (DAF) and CD59 in gastrointestinal tumours. Eur. J. Cancer. 35:117-124.

16. Thorsteinsson, L., O’Dowd, G.M., Harrington, P.M., and Johnson, P.M. 1998. The complement regulatory proteins CD46 and CD59, but not CD55, are highly expressed by glandular epithelium of human breast and colorectal tumour tissues. APMIS. 106:869-878.

17. Jain, R.K. 1991. Haemodynamic and transport barriers to the treatment of solid tumours. Int. J. Radiat. Biol. 60:85-100.

18. Galan, J.E., and Collmer, A. 1999. Type III secretion machines: bacterial devices for protein delivery into host cells. Science. 284:132-138. 